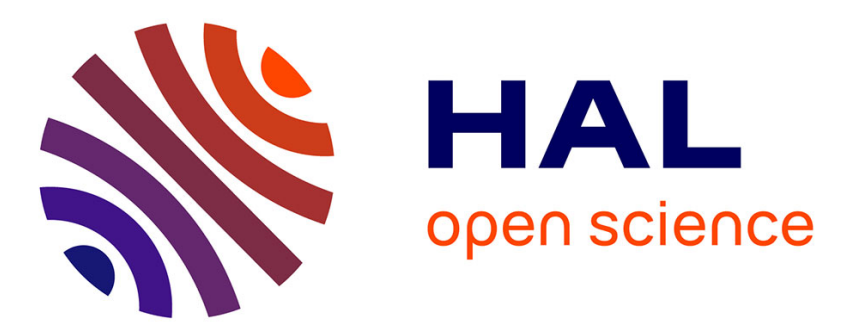

\title{
Average-based Adaptive Grid Refinement in Hybrid LES
}

S. Mozaffari, Michel Visonneau, Jeroen Wackers

\section{To cite this version:}

S. Mozaffari, Michel Visonneau, Jeroen Wackers. Average-based Adaptive Grid Refinement in Hybrid LES. Direct and Large-Eddy Simulation XII, ERCOFTAC Series 27, 2020. hal-02613463

\section{HAL Id: hal-02613463 \\ https://hal.science/hal-02613463}

Submitted on 20 May 2020

HAL is a multi-disciplinary open access archive for the deposit and dissemination of scientific research documents, whether they are published or not. The documents may come from teaching and research institutions in France or abroad, or from public or private research centers.
L'archive ouverte pluridisciplinaire HAL, est destinée au dépôt et à la diffusion de documents scientifiques de niveau recherche, publiés ou non, émanant des établissements d'enseignement et de recherche français ou étrangers, des laboratoires publics ou privés. 


\title{
Average-based Adaptive Grid Refinement in Hybrid LES
}

\author{
S. Mozaffari, M. Visonneau, and J. Wackers
}

\section{Introduction}

Hybrid LES models were proposed as a solution to the high computational costs of Large Eddy Simulation (LES) for complex geometries at high Reynolds numbers. To take advantage of their capabilities, meshes with sufficient resolution around the turbulent regions of simulation are necessary. Adaptive Grid Refinement (AGR) creates these meshes by starting the solution on a coarse grid and refining this grid locally to accurately resolve areas of interest. This is an ideal way to efficiently solve flow problems that have strong local structures whose position is not known a priori, without excessive computational effort.

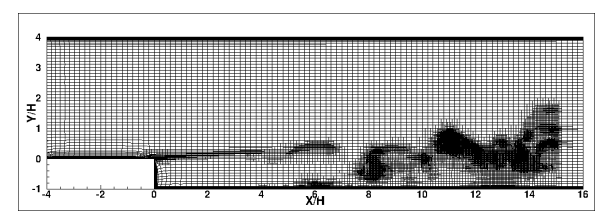

Fig. 1 Refined mesh based on instantaneous solutions (AGR-100, see Table 1).

In hybrid LES flows which are highly unsteady, following all the structures of the turbulent flow with AGR (Figure 1) means that the mesh is modified every few time steps. Unfortunately, this introduces extra truncation errors due to the interpolation which is performed during the grid refinement in order to transfer the solution between grids. To avoid these rapid changes in the adapted mesh, the refinement criterion which determines which part of the mesh should be refined, could be based on

S. Mozaffari · M. Visonneau $\cdot$ J. Wackers

LHEEA, Centrale Nantes/CNRS, 44300 Nantes, France, e-mail: \{sajad.mozaffari, michel.visonneau, jeroen. wacker\} dec-nantes. fr 
the averaged quantities instead of the instantaneous solutions. Thus, the refinement is restricted to capturing the large energy-containing eddies, without following all the turbulent structures. Toosi and Larsson [3] also tested an averaged error estimator for grid adaptation in LES simulation.

In this paper, AGR with averaged criteria is tested for the backward-facing step flow of Vogel and Eaton [4]. Improved Delayed Detached-Eddy Simulation (IDDES) [2] is used in the ISIS-CFD solver, developed at Centrale Nantes/CNRS.

\section{Adaptive Grid Refinement with Averaged Quantities}

The AGR algorithm [5] begins with the computational domain covered with a coarse grid. As the calculation progresses, the mesh is regularly adapted based on the refinement criterion: a tensor field computed from the second derivatives of the solution, whose values determine which parts of the mesh should be refined or derefined. The global mesh size is imposed by a threshold parameter $T_{r}$ which indicates the desired cell size.

To limit the mesh evolution, a criterion is desired which evolves slowly in time. Therefore it is based on a time-averaging process, either by averaging the instantaneous flow field or the computed criterion. In the first case, a time-averaged solution is computed, the refinement criterion is defined based on this averaged solution, and the mesh is adapted accordingly. In the second case, the criterion is computed every time step from the instantaneous solutions and the refinement is based on the average of the computed criteria.

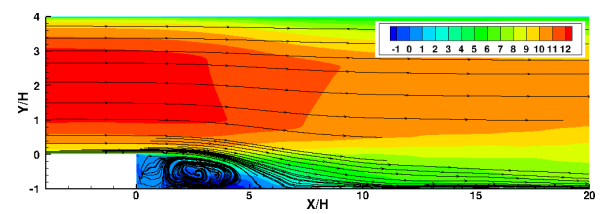

Fig. 2 Average axial flow and streamlines for the BFS (Fine grid, see Table 1).

\section{Backward-Facing Step Simulation}

The backward-facing step (BFS) studied here is based on [4, 2, 1]. Behind the step, the flow has three main regions (Figure 2): the shear layer region with the recirculation, the reattachment zone, and the relaxation region where the turbulence spreads through the entire channel. The computational domain based on the step height $(H)$, runs from $x=-4 H$ to $x=20 H$ in stream-wise direction, with size $4 H$ span-wise direction and channel expansion ratio is $5 / 4$. 
At the inlet, velocity and turbulence from a precursor RANS calculation are imposed. In ISIS-CFD, periodic boundary condition is not available with AGR, so mirror boundary conditions are specified in span-wise direction. A no-slip condition is specified on the solid walls with $y^{+}=0.5$ and on the outlet boundary a frozen pressure is applied. The Reynolds number based on the step height, $H=0.038 \mathrm{~m}$, is equal to 28,000 and the non-dimensional time step is 0.018 , for 10,000 time steps in total.

\section{Results and Discussion}

Several averaging strategies (Table 1) are tested and compared in terms of recirculation length, wall shear stress (Figure 3) and turbulent structures (Figure 7). Reference computations are performed on a fine fixed mesh without AGR, and by AGR without averaging (AGR-100), with refinement every two time steps, unlike the average-based computations that have refinement every 25 time steps.

While the Fine mesh works well, AGR-100 creates perturbations in the skin friction on the lower wall. Although AGR follows the turbulent structures and refines the mesh where unsteadiness is high (Figure 7), the frequent mesh changes perturb the solution. This confirms the interest of average-based refinement.

Table 1 Description of the computations, with computed recirculation length.

\begin{tabular}{|c|c|c|c|c|c|}
\hline Computation & Average method & Threshold $T_{r}$ & Cells $\left(\times 10^{6}\right)$ & $L_{r}(X / H)$ & Error (\%) \\
\hline Exp. & - & - & - & 6.66 & - \\
\hline Fine mesh & - & - & 9.5 & 6.5 & -2.5 \\
\hline AGR-100 & No averaging & 0.01 & 5.5 & 11 & 65.2 \\
\hline CA@-25 & Avg. flow field & 0.0025 & 2.4 & 7.14 & 7.2 \\
\hline AC@-25 & Avg. criterion & 0.0025 & 1.3 & 7.9 & 18.7 \\
\hline AC@-20 & Avg. criterion & 0.0020 & 2.5 & 7.0 & 5.1 \\
\hline AC@-15 & Avg. criterion & 0.0015 & 9.9 & 6.9 & 3.6 \\
\hline $\mathrm{AC} @-25 \mathrm{~B}^{\dagger}$ & Avg. criterion & 0.0025 & 2.0 & 6.5 & -2.5 \\
\hline
\end{tabular}

\subsection{Average of the Flow Fields (CA)}

For CA@-25 the criterion is based on the averaged flow. The final mesh in Figure 4 shows refinement around the free shear layer, but the refined mesh is not regular. This is because a part of the average solution was interpolated from the original mesh to the refined mesh. Due to the linear interpolation used, this contribution to the average solution is not smooth enough to allow the computation of a secondderivative refinement criterion.

For other parts of the domain the mesh remains coarse especially behind the step (recirculation region) where the velocity gradients are low. Figure 3 shows an 

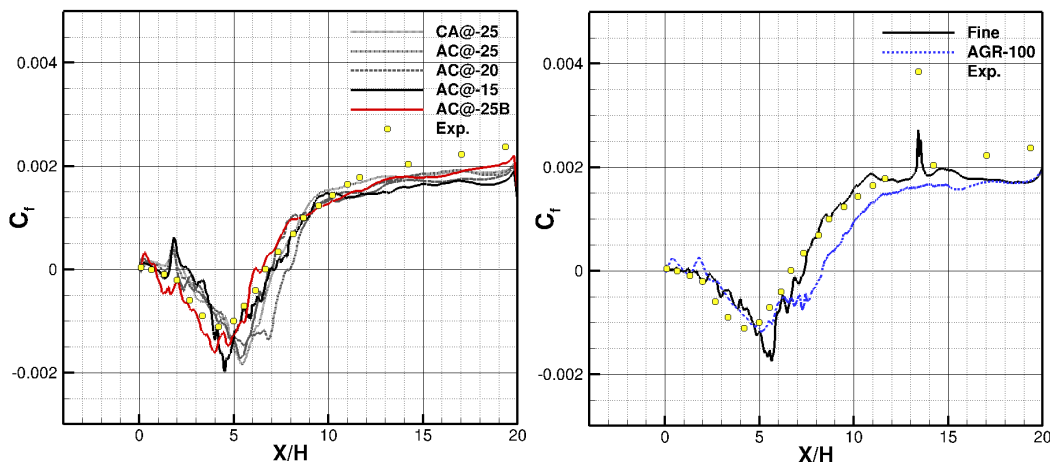

Fig. 3 Mean friction coefficients along the lower wall, for average-based (left) and reference (right) computations - CA: average of the flow fields, and AC: average of the criterion.
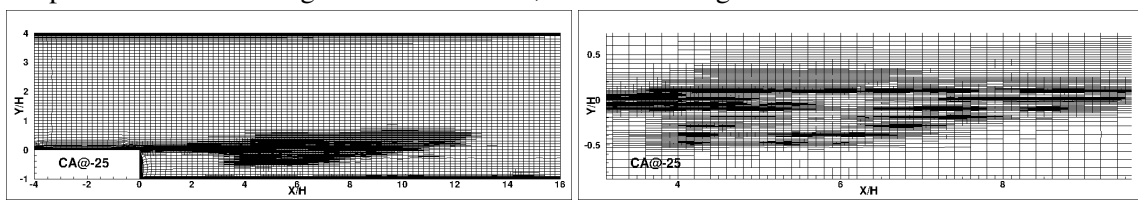

Fig. 4 Final refined mesh for the time-averaged flow field (CA@-25), with mesh detail.

overestimation for the mean friction coefficient distribution before the reattachment point, while the distance to the step tip (reattachment length) for this case is more than $7 \%$ larger compared to the experiment. Thus, the AGR based on the averaged flow fields, with some degree of error, can predict the behavior of the flow, but the irregular refinement makes the mesh invalid.

\subsection{Average of the Criterion (AC)}

For AC@-25, the averaging is applied over the computed criteria. For the same threshold value as the previous case, the final mesh is coarser (Table 1), so the difference between the computed mean friction coefficient and the experimental data increases (Figure 3). However, the mesh is of better quality (Figure 5).

Threshold Effect. Since the AC@-25 mesh is too coarse to capture the flow details, the threshold which globally indicates the requested amount of mesh refinement, is lowered to $T_{r}=0.0020$ and 0.0015 . As the threshold is decreased, larger regions of the domain are refined (Figure 5) and the final mesh converges to a larger number of cells. This increase in the refinement occurs mostly around the shear layer and the reattachment region. The AGR also captures the edges of the attached boundary layers.

Using lower thresholds improves the solutions, particularly the wall shear stress in the reattachment region (Figure 3). Table 1 shows that for the lowest threshold 


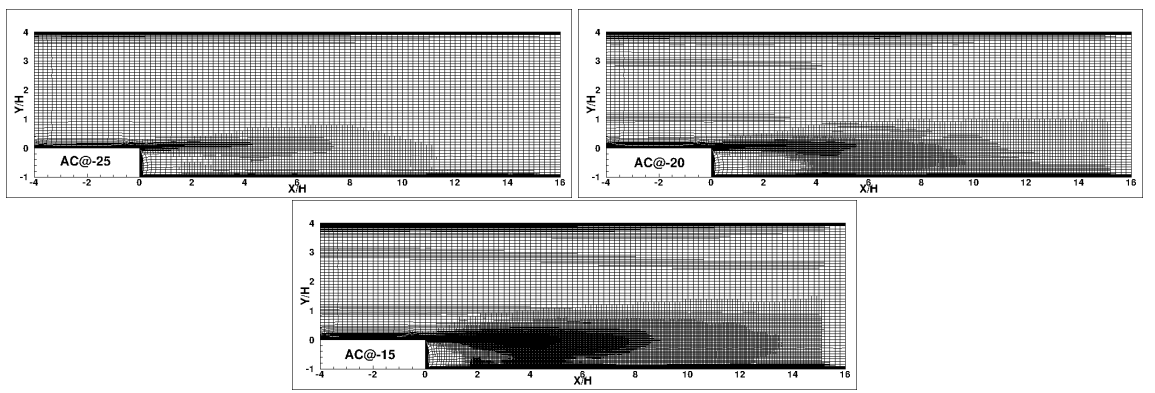

Fig. 5 The final refined meshes for the computations with time-averaged criterion.

$\left(T_{r}=0.0015\right)$ the difference between computed reattachment lenght and the experiment decreased to $3.6 \%$.

Influence of the Initial Mesh. In none of the previous cases, the mesh is refined in the recirculation zone. In the LES simulation of a BFS by Toosi and Larsson [3], adaptive refinement did not refine the grids in the recirculation zone as well. However, secondary vortices established in the recirculation zone can perturb the shear layer and might have an effect on the downstream flow. Therefore, the computation AC@-25B is performed using an initial mesh with a refinement box behind the step (Figure 6). The other settings are as for AC@-25.

By using the refinement box, more turbulent structures are captured and a larger region is refined compared to AC@-25. The wall shear stress in the recirculation region is improved and the reattachment length error reduced to less than 3\%. Figure 7 shows that, compared with other AGR cases, more turbulent structures in the recirculation zone are captured and the free-shear layer is represented better. These improvements could be the result of the small vortices in the recirculation zone which are captured thanks to the refinement box.

\section{Conclusion}

Adaptive grid refinement was coupled with a hybrid LES turbulence model to simulate the flow over a backward facing step. Using AGR with a refinement criterion based on the averaged solution is ineffective, since this solution is not smooth enough to allow the evaluation of its second derivative. However, using the averaged result of the computed criterion at each time step gives an advantage over AGR with frequent refinements without averaging, in terms of the accuracy of the solutions and also the computational cost. By reducing the threshold sufficiently, an accurate solution in the reattachment and relaxation regions is obtained. However, the refinement box in the recirculation zone shows that turbulent structures which are too small to create adaptive refinement with any criterion tested, may have an influence on the solution. How to detect and adaptively refine such zones, is an open question. 
S. Mozaffari, M. Visonneau, and J. Wackers

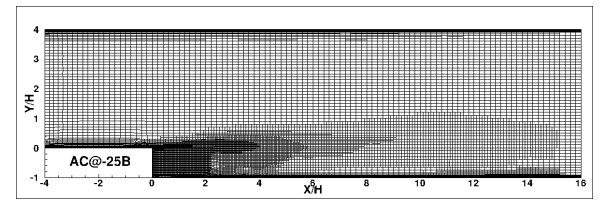

Fig. 6 The effect of the refinement box $x \in[0,2], y \in[-1,0]$ in the recirculation zone.

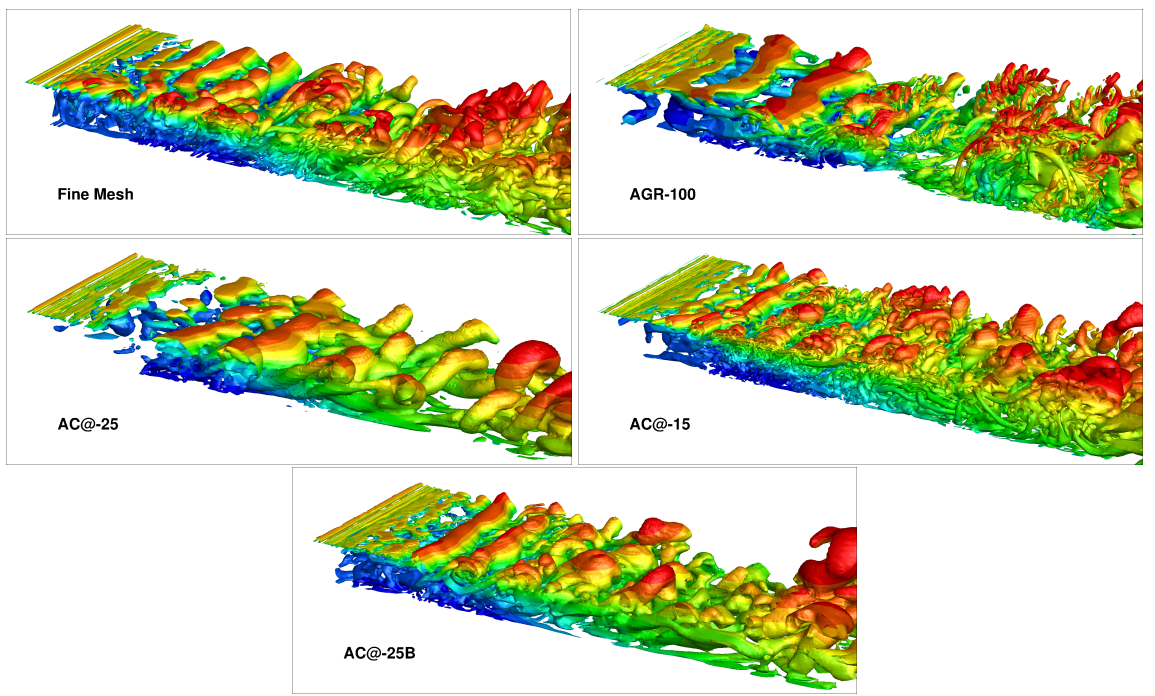

Fig. 7 Iso-surface second invariant $Q=2500$; colored by averaged velocity $\mathrm{X}$.

\section{References}

1. Gritskevich, M.S., Garbaruk, A.V., Schütze, J., Menter, F.R.: Development of DDES and IDDES Formulations for the k- $\omega$ Shear Stress Transport Model. Flow, Turbulence and Combustion. 88(3), 431-449 (2012).

2. Shur, M.L., Spalart, P.R., Strelets M.KH., Travin, A.K.: A hybrid RANS-LES approach with delayed-DES and wall-modelled LES capabilities. Int. J. Heat and Fluid Flow. 29(6), 16381649 (2008).

3. Toosi, S., Larsson, J.: Anisotropic grid-adaptation in large eddy simulations. Computers \& Fluids. 156, 146-161 (2017)

4. Vogel, J.C., Eaton, J.K.: Combined heat transfer and fluid dynamic measurements downstream of a backward-facing step. J. Heat Transfer. 107(4), 922-929 (1985).

5. Wackers, J., Deng, G.B., Guilmineau, E., Leroyer, A., Queutey, P., and Visonneau, M.: Combined refinement criteria for anisotropic grid refinement in free-surface flow simulation. Comput. Fluids. 92, 209-222 (2014). 